\title{
Impact sensing headgear-a physical approach to provide critical information
}

\begin{abstract}
We report on the development of impact sensing technology in the form of strips mounted in a headband or cap positioned around the skull, to be used for rapidly evaluating the likelihood of a concussion. The technology reported here is designed to produce an image on the sensing strip at the location of the impact, with the image itself being related to the magnitude of the impact. In contrast to accelerometer-based impact detection, the technology reported here creates a permanent marking that is proportional to force and bypasses the complexities of embedded electronics.
\end{abstract}

Volume 2 Issue 4 - 2017

\author{
Marcos Dantus, Gary J Blanchard \\ Department of Chemistry, Michigan State University, USA
}

Correspondence: Gary J Blanchard, Michigan State University, Department of Chemistry, East Lansing, MI 48824, MTBI sense LLC, Okemos, USA, Email blanchard@chemistry.msu.edu

Received: April 14, 2017 | Published: May 05, 2017

\section{Introduction}

The significance of concussive injury prevention in sports is becoming more obvious as knowledge is acquired regarding longerterm outcomes. Among the factors that have led to increased concern is the length of time required for the effects of concussive injury to manifest themselves. A traumatic head blow occurs in milliseconds and symptoms of concussion may appear hours later, yet return to play decisions need to be made in seconds. The detection of concussion is, in many cases, challenging. Medical professionals perform such evaluations based on player neurological and cognitive response, sometimes evaluated using standardized evaluation protocols, such as the $\mathrm{SCAT}^{1}{ }^{1}$ or MACE${ }^{2}$ tests. Significant challenges in the evaluation of concussive injury include the sometimes delayed onset of symptoms and the availability of medical personnel to perform such evaluations immediately following impact. There is thus an urgent need to provide tangible proof to medical professionals and other responsible personnel of whether or not a player experienced an impact to the head and, if so, where the impact occurred and the magnitude of the impact. If such information were widely available, it could help minimize the occurrence of "second impact syndrome", which is thought to be a serious problem due to the injured brain's diminished ability to withstand a subsequent injury. ${ }^{3-5}$

With these concerns in mind we propose and demonstrate impact sensing technology that has been designed to satisfy several criteria: (a) wide accessibility, (b) output that is easy to understand by nonmedical personnel, and (c) reliability. A key focus in this effort was to detect force rather than acceleration alone. The impact sensing technology reported here is present in the form of thin strips that are mounted in a headband or cap and that can be removed easily for inspection and/or replacement. The strips record magnitude and location of head impact experienced by the wearer, with the mechanism of force recording being the response of a proprietary multi-level thin film sensing assembly. ${ }^{6,7}$ We have demonstrated the proportional relationship between magnitude of impact and sensor response using calibrated test equipment in our laboratory. Before discussing the results for our force-recording sensors, it is useful to place these results in the context of other technology that is in wide use. Accelerometer-based sensing with the device affixed to the athlete either on the cranium or in the mouth is in current use. The results of impact sensing from these technologies vary with the location and specific properties of the device tested, and in all cases respond to acceleration rather than force. ${ }^{8}$ In addition, much of the presently available, accelerometer-based devices rely on wireless transmission of impact events, whereas the force-recording strips reported here do not transmit data wirelessly, and provide information specific to the point of impact.

\section{Experimental methods}

\section{Impact sensing strips}

The impact sensing strips reported here (Figure 1) are designed to produce a physical image that is proportional to the magnitude and location of the impact experienced. The strips are designed to produce responses that vary over the relevant range of forces experienced (expressed as $\mathrm{g}$ forces). The design is proprietary. ${ }^{6,7}$

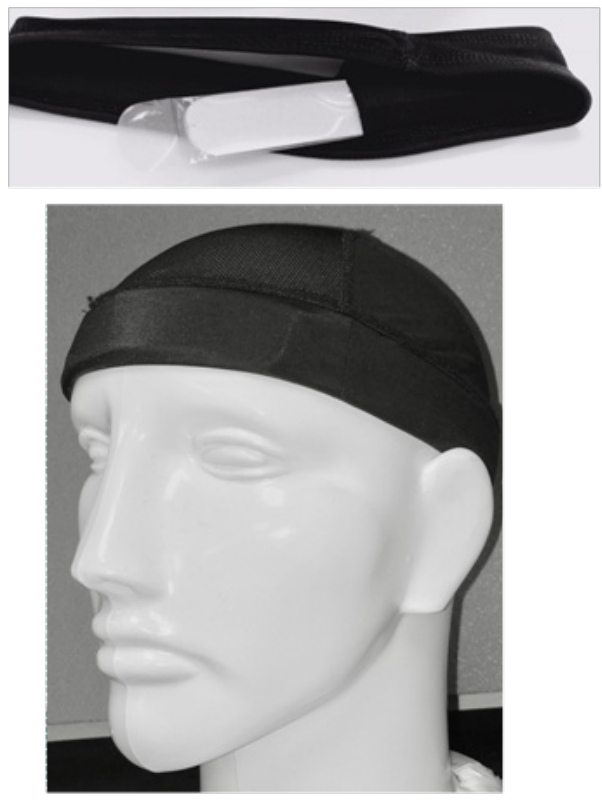

Figure I (Top) Impact sensing strip shown partially removed from head band. (Bottom) cap mounted on a mannequin head.

\section{Sensor calibration}

A laboratory grade accelerometer (PCB Piezoelectronics, Model $352 \mathrm{C} 03$ ) was attached to a mannequin head (human head weight, 
ca. $5 \mathrm{~kg}$ ), which was mounted on a flexible lever arm. Initial tests on inexpensive accelerometers were unreliable, and the use of such accelerometers was discontinued. The impact experienced by the mannequin head was controlled by the height from which it was dropped and the force was recorded by the accelerometer and associated electronics and stored on a pc. Software for accelerometer signal acquisition was written in-house using National Instruments LabVIEW ${ }^{\circledR}$ software. Tests were performed on the mannequin under both helmeted and non-helmeted conditions.

\section{Results and discussion}

Impact force is experienced by a player exposed to potentially concussive injury. Within criterion (a) listed above, affordability and utility for both helmeted and non-helmeted sports were considered. For criterion (b), it is important to provide information that is easy to understand and to have that information available at the time when it is needed most-immediately after the potentially concussive injury occurs. For criterion (c) it is important that the device not fail unexpectedly (i.e. false negative), or produce signals based on acceleration alone (i.e. false positive).

Criterion (a) was satisfied by incorporation of the impact sensing strips into the headgear. The use of headbands or caps is not restricted to any particular sport and is appropriate for use in either helmeted or non-helmeted sports. To satisfy criterion (b), the response of the strips (Figure 2) was designed to produce images that vary in proportion to the magnitude of the impact, with a faint circle or portion thereof representing mild impact, a solid circle representing moderate impact and a solid circle surrounding a star representing high to severe impact. The forces associated with the images produced are indicated in Figure 2. The goal in designing the functional form of the sensor response was to provide relative ranges of impact. Focusing on a specific amount of force is not appropriate for the intended purpose of the sensor because the device is not a concussion detector. A concussion can only be diagnosed by a trained medical professional and the sensors are intended to function as an extension of the clinical examination. In addition, each potentially concussive injury is unique owing to the combination on individual physiology and the detailed mechanics of the impact associated with the injury. The primary objective of these sensors is to provide information to any sideline personnel indicating whether or not a player should seek medical attention.

In addition to their primary use as head impact sensors, these devices can be used in the evaluation of athletic equipment. We have studied the relationship between the force (as measured by sensor strip and corroborated with an accelerometer) experienced by a football helmet and the force experienced by the mannequin head on which the helmet was mounted. The data (Figure 3) demonstrate that the relationship between force experienced by the helmet and that transferred to the head can differ, depending on the magnitude of the impact. Before describing the physical basis for these data we hasten to point out that their functional form can vary with brand and model of helmet and these data are intended to illustrate the capabilities of the sensing strips rather than as an evaluation of a specific helmet type.

The data shown in Figure 3 can be understood as follows. For low impact, the helmet and mannequin head experience the same deceleration on impact because the padding in the helmet is sufficiently rigid that it is not distorted by the force of impact. In the mid-range of forces, there is a measureable difference between force experienced by the helmet and the mannequin head, reflecting the helmet padding responding to the force and cushioning the mannequin head. For high impact forces of 80-100 g, the impact force experienced by the helmet and mannequin head become similar, owing to the helmet padding reaching the limit of its compliance and transmitting all of the force to the mannequin head. We expect that many factors, including helmet design, age and use history influence the functional form of these data. It is significant to note that, within the compliance range of the padding, the reduction in force experienced by the head is approximately constant.
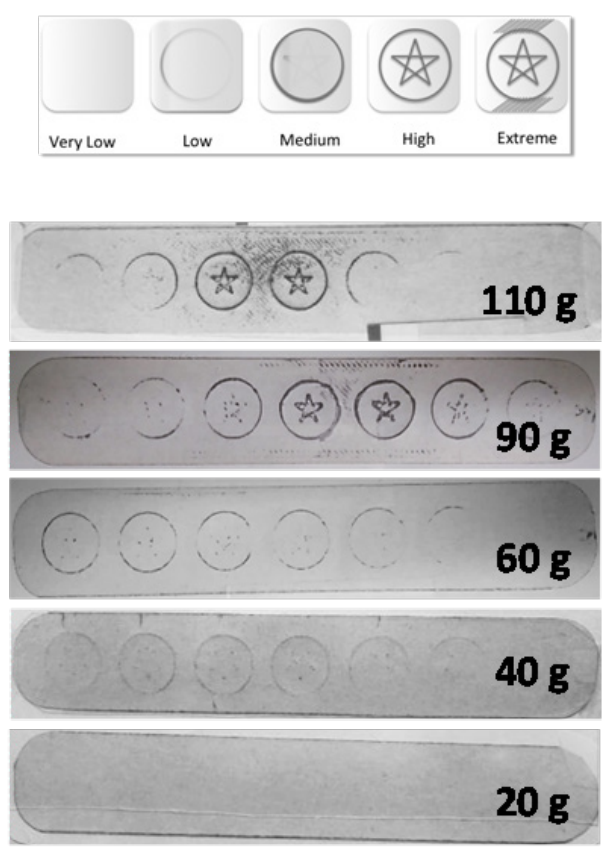

Figure 2 (Top) Schematic of sensor response as a function of impact force experienced. (Bottom) actual sensor images after having experienced impact while mounted to mannequin head covered with football helmet. Readings on the right side of the strips are accelerometer readings associated with the impacts.

These sensors have already been used by football as well as male and female soccer players at certain games and practices at Michigan State University. We have found a reasonable correlation between high impact force marks and diagnosed concussions. This information is preliminary given the small numbers involved. Data analytics following the widespread use of these sensors could lead to the development of a statistical relationship between the magnitude and/or location of impact and the likelihood of a concussion well before concussion symptoms appear. The technology reported here should help minimize the occurrence of second concussion syndrome, especially in youth leagues that lack medical personnel to administer concussion evaluation on the sidelines.

\section{Conclusion}

We have reported on the design and demonstration of head impact sensing strips, mounted in headgear and intended to be worn during activities in which potentially concussive impacts could be expected. These sensors are physical, as opposed to electronic, in nature and respond to impact force rather than acceleration. We have calibrated the sensor response and dynamic range in the laboratory and demonstrate the utility of these devices in the context of the data presented in Figure 3. As noted in the introduction, the goal of this work was to 
demonstrate sensing technology that satisfies the constraints of broad availability, intuitively understood data output and high reliability.

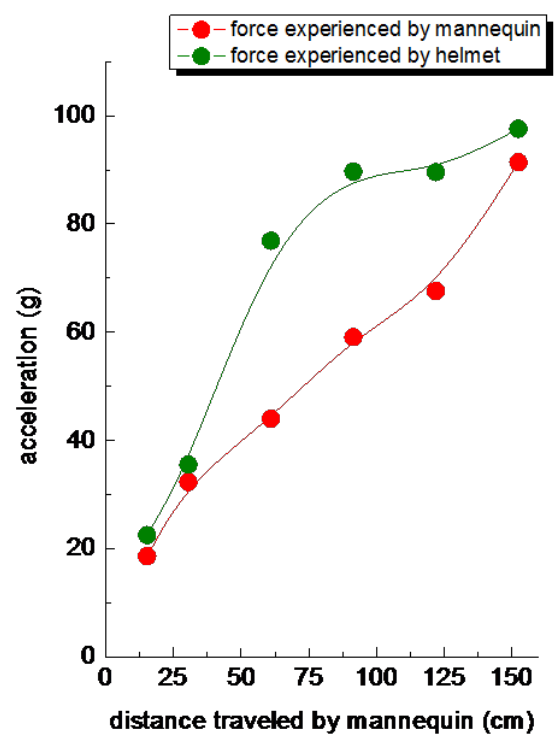

Figure 3 Relationship between sensor force response (corroborated by accelerometer readings, indicated on $y$-axis) and distance travelled by helmeted mannequin head before impact. Red data points represent force experienced at the mannequin head. Green data points represent force experienced at the helmet outer surface.

\section{Acknowledgements}

None.

\section{Conflict of interest}

The author declares no conflict of interest.

\section{References}

1. Mc Crory P, Meeuwisse W, Aubry M, et al. Consensus statement on concussion in sport: the $4^{\text {th }}$ International Conference on concussion in Sport held in Zurich, November 2012. British Journal of Sports Medicine. 2013;47:259-262.

2. Military acute concussion evaluation; 2012.

3. Cantu RC. Second-Impact Syndrome. Clin Sports Med. 1998;17(1):3744.

4. Mc Crory PR, Berkovic SF. Second impact syndrome. Neurology. 1998;50(3):677-683.

5. Bey T, Ostick B. Second Impact Syndrome. West J Emerg Med. 2009;10(1):6-10.

6. Dantus M, Blanchard G, Blanchard S. Materials with detectable compression memory. 2016;62:26-765.

7. Dantus M, Blanchard G, Osborn DI, et al. Materials and apparatus with multiple impact level and torque detection. 2017;15:164-996.

8. Wu LC, Nangia V, Bui K, et al. In Vivo Evaluation of wearable head impact sensors. Ann Biomed Eng. 2016;44(4):1234-1245. 